\title{
Time-resolved structural biology over longer reactions and including complementary methods, but with less sample
}

\author{
Allen M. Orville et al \\ Principal Scientist, XFEL Hub at Diamond \\ Diamond Light Source, Harwell Science and Innovation Campus \\ Didcot, Oxfordshire OX11 0DE \\ United Kingdom
}

X-ray free electron lasers (XFEL) sources enable new science, but require novel sample delivery methods that exploit the pulse sequence of the particular source, but do not waste sample between XFEL pulses. We developed acoustic droplet ejection (ADE) as a general, touchless, on-demand method that uses focused sound waves to eject picoliter to nanoliter volume droplets from one place to another. In our first applications, we collected SFX datasets from droplets launched in synchrony to intersect each XFEL pulse (Roessler et al 2016). We have also coupled ADE methods with a conveyor belt drive to enable timeresolved SFX (tr-SFX) at the LCLS. Our system is optimized for crystallography and X-ray emission spectroscopy (XES) measurements of photochemical reactions over a wide-range of time scales and illumination schemes (Young, Ibrahim, and Chatterjee et al 2016; Fuller and Gull et al 2017). Indeed, the combination of tr-SFX + XES, wherein both types of data are obtained from the same sample and X-ray pulse, provides important complementary information that impacts mechanistic insights. We are also exploiting the region on the belt between the ADE transducer and the X-ray interaction point to introduce additional experimental perturbations that initiate a range of enzyme reactions. I will discuss the potential impact to tr-SFX studies of many macromolecules operating under physiological temperature and pressure.

Supported in part by grant 102593 from the Wellcome Trust and the BBSRC

Christian G. Roessler, Rakhi Agarwal, Marc Allaire*, Roberto Alonso-Mori, Babak Andi, José F. R. Bachega, Martin Bommer, Aaron S. Brewster, Michael C. Browne, Ruchira Chatterjee, Eunsun Cho, Aina E. Cohen, Matthew Cowan, Sammy Datwani, Victor L. Davidson, Jim Defever, Brent Eaton, Richard Ellson, Yiping Feng, Lucien P. Ghislain, James M. Glownia, Guangye Han, Johan Hattne, Julia Hellmich, Annie Héroux, Mohamed Ibrahim, Jan Kern, Anthony Kuczewski, Henrik T. Lemke, Pinghua Liu, Lars Majlof, William M. McClintock, Stuart Myers, Silke Nelsen, Joe Olechno, Allen M. Orville", Nicholas K. Sauter, Alexei S. Soares", S. Michael Soltis, Heng Song, Richard G. Stearns, Rosalie Tran, Yingssu Tsai, Monarin Uervirojnangkoorn, Carrie M. Wilmot, Vittal Yachandra, Junko Yano, Erik T. Yukl, Diling Zhu, Athina Zouni; “Acoustic injectors for drop-on-demand serial femtosecond crystallography" (2016) Structure 24, 631-640

Iris D. Young, Mohamed Ibrahim, Ruchira Chatterjee, Sheraz Gul, Franklin Fuller, Sergey Koroidov, Aaron S. Brewster, Rosalie Tran, Roberto Alonso-Mori, Thomas Kroll, Tara Michels-Clark, Hartawan Laksmono, Raymond G. Sierra, Claudiu A. Stan, Rana Hussein, Miao Zhang, Lacey Douthit, Markus Kubin, Casper de Lichtenberg, Long Vo Pham, Håkan Nilsson, Mun Hon Cheah, Dmitriy Shevela, Claudio Saracini, Mackenzie A. Bean, Ina Seuffert, Dimosthenis Sokaras, Tsu-Chien Weng, Ernest Pastor, Clemens Weninger, Thomas Fransson, Louise Lassalle, Philipp Bräuer, Pierre Aller, Peter T. Docker, Babak Andi, Allen M. Orville, James M. Glownia, Silke Nelson, Marcin Sikorski, Diling Zhu, Mark S. Hunter, Andy Aquila, Jason E. Koglin, Joseph Robinson, Mengning Liang, Sébastien Boutet, Artem Y. Lyubimov, Monarin Uervirojnangkoorn, Nigel W. Moriarty, Dorothee Liebschner, Pavel V. Afonine, David G. Waterman, Gwyndaf Evans, Philippe Wernet, Holger Dobbek, William I. Weis, Axel T. Brunger, Petrus H. Zwart, Paul D. Adams, Athina Zouni, Johannes Messinger, Uwe Bergmann, Nicholas K. Sauter, Jan Kern, Vittal K. Yachandra, Junko Yano, "Structure of photosystem II and substrate binding at room temperature" (2016) Nature 540, 453-457

Franklin D. Fuller, Sheraz Gul, Ruchira Chatterjee, Ernest S. Burgie, Iris D. Young, Hugo Lebrette, Vivek Srinivas, Aaron S. Brewster, Tara Michels-Clark, Jonathan A. Clinger, Babak Andi' Mohamed Ibrahim, Ernest Pastor, Casper de Lichtenberg, Rana Hussein, Christopher J. Pollock, Miao Zhang, Claudiu A. Stan, Thomas Kroll, Thomas Fransson, Clemens Weninger, Markus Kubin, Pierre Aller, Louise Lassalle, Philipp Bräuer, Muhamed Amin, Sergey Koroidov, Christian G. Roessler, Marc Allaire, Raymond G. Sierra, Peter T. Docker, James M. Glownia, Silke Nelson, Jason Koglin, Diling Zhu, Matthieu Chollet, Sanghoon Song, Henrik Lemke, Mengning Liang, Dimosthenis Sokaras, Roberto Alonso-Mori, Athina Zouni, Johannes Messinger, Uwe Bergmann, Amie K. Boal, J. Martin Bollinger, Jr., Carsten Krebs, Martin Högbom, George N. Phillips, Jr., Richard D. Vierstra, Nicholas K. Sauter, Allen M. Orville, Jan Kern, Vittal K. Yachandra, Junko Yano "Drop-on-Demand Sample Delivery for Studying Biocatalysts in Action at XFELs" (2017) Nature Methods (in press) doi:10.1038/nmeth.4195 